Inga Vermeulen, Georg Brun, Christoph Baumberger

\title{
Five Ways of (not) Defining Exemplification
}

\begin{abstract}
The notion of exemplification is essential for Goodman's theory of symbols. But Goodman's account of exemplification has been criticized as unclear and inadequate. He points out two conditions for an object $\mathrm{x}$ exemplifying a label $\mathrm{y}$ : (C1) y denotes $\mathrm{x}$ and $(\mathrm{C} 2) \mathrm{x}$ refers to $\mathrm{y}$. While $(\mathrm{C} 1)$ is uncontroversial, $(\mathrm{C} 2)$ raises the question of how "refers to" should be interpreted. This problem is intertwined with three further questions that consequently should be discussed together with it. Are the two necessary conditions $(\mathrm{C} 1)$ and $(\mathrm{C} 2)$ conjointly sufficient? Do they amount to a definition of "exemplification"? Which notions of Goodman's theory are basic, and hence undefined? In this paper, we address these questions and defend a reconstruction of the notion of exemplification that interprets "refers to" in (C2) as exemplificational reference and hence treats "exemplification" as a basic notion of Goodman's theory. Firstly, we argue that even though the notion of exemplification is not defined, it is still sufficiently clear. This ensures its contribution to Goodman's theory of symbols. Secondly, we show that our account is plausible as an interpretation of Goodman's and Elgin's writings, although it implies that some of Goodman's theorems about selfreference have to be weakened. Thirdly, we argue that it is the only materially adequate reconstruction of Goodman's notion of exemplification, whereas the alternative definitional accounts fail.
\end{abstract}

In his theory of symbols, Nelson Goodman uses "reference" as a very broad notion covering all sorts of symbolization, all cases of "standing for". He neither attempts to define "reference" nor to explain how referential relationships are established. Rather, he distinguishes and compares different forms of reference. There are at least two fundamental ones, denotation and exemplification. Denotation is reference from a symbol to one or many objects it applies to. Thus, a name denotes its bearer and a predicate each object in its extension. Denoting symbols are called labels. Labels do not have to be verbal. A portrait denotes its subject

We would like to thank Catherine Z. Elgin for helpful comments on an earlier draft. 
and a music-score the performances complying with it. Exemplification runs in the opposite direction and is reference from an object back to a label that applies to it. Symbols that exemplify are called samples. Thus, a chip of paint on a manufacturer's sample card exemplifies, in normal use, a colour-label that applies to it and a tone produced by the concert-master before the performance a label which denotes its pitch. Importantly, no object is in itself a label or a sample, but only if used as part of a symbol system. In particular, this implies that a symbol is always a member of a scheme; that is, a set of alternative symbols, that is correlated with a realm, which is a set of objects to which at least one of the symbols refers.

The notion of exemplification is characteristic of and essential for Goodman's theory of symbols. Goodman introduces it in Languages of Art $(L A, \mathrm{II})$ and uses it to explain artistic expression as well as the symbolic functions of fictional labels, metaphors, abstract paintings and other nondenotational symbols. It furthermore serves as a basis for analysing important parallels and contrasts between these symbolic functions and the role samples play in the sciences. But Goodman's account of exemplification is not without problems. His writings raise questions of interpretation and his account has been criticized as unclear and inadequate. However, giving up the notion of exemplification would be devastating for Goodman's theory, for it would severely reduce its range of application and its explanatory power. In this paper, we suggest a reconstruction of the notion of exemplification that is materially adequate as well as sufficiently clear to secure the contribution of this notion to Goodman's theory of symbols. Since this theory was systematized and further developed by Catherine Elgin (especially in 1983), we rely both on Goodman's and Elgin's writings.

Section 1 of this paper gives an overview of possible interpretations of "exemplification" that remain in a broadly Goodmanian framework and presents our reconstruction of the notion of exemplification from a purely systematic point of view. We then discuss in section 2 the relevant passages in the writings of Goodman and Elgin and argue that our reconstruction gives a plausible interpretation. Finally, in section 3 we analyse the debate on the notion of exemplification and show that our 
reconstruction is the only materially adequate one of the proposals considered.

\section{Reconstructing the notion of exemplification}

Goodman points out two conditions for something being a sample of a given label. It must be denoted by the label and it must refer to the label (LA, 52; 53;MM, 59):

(1) There are two conditions for $\mathrm{x}$ exemplifying $\mathrm{y}$ : (C1) y denotes $\mathrm{x}$ and $(\mathrm{C} 2) \mathrm{x}$ refers to $\mathrm{y}$.

\subsection{Two necessary conditions}

According to condition (C1), exemplification presupposes denotation in the opposite direction. The paint chip on the manufacturer's sample card can be used as a sample of "off-white" only if this label applies to, that is, denotes the chip. But an object does not exemplify all labels that apply to it. Maybe "rectangular" and "manufactured by a well known company" apply to the chip, but those labels are not exemplified if the chip is used in the usual way. Consequently, exemplification cannot be identified with the converse of denotation, but only with a certain subrelation thereof. Condition (C1) is thus necessary but not sufficient for exemplification. Only if $\mathrm{y}$ denotes $\mathrm{x}$, may $\mathrm{x}$ exemplify $\mathrm{y}$. However, although exemplification presupposes denotation in opposite direction, exemplification is reference in one direction only, namely from sample to label.

Condition (C2) additionally requires that an object refers to the label it exemplifies. The paint chips do not exemplify all the labels they instantiate, but only those they refer to. In normal use, these are colourlabels. However, it is not clear how exactly this second condition should be interpreted. The primary question is how one should interpret "refers to" in (C2). This problem is intertwined with three further questions. Firstly, are the two necessary conditions $(\mathrm{C} 1)$ and (C2) sufficient, if taken together? That is, can (1) be read as (1.1)?

(1.1) $\mathrm{x}$ exemplifies $\mathrm{y} \leftrightarrow \mathrm{y}$ denotes $\mathrm{x} \wedge \mathrm{x}$ refers to $\mathrm{y}$. 
Secondly, do the two conditions (C1) and (C2) taken together amount to a definition of "exemplification", such that the biconditional in (1.1) could be replaced by " $=$ df"? Even though Goodman's semantics is extensional, it does not eliminate the difference between a true biconditional and a definition, since coextensive expressions can differ in secondary extensions. ${ }^{1}$ Thirdly, there is the question of which notions are basic and therefore undefined in Goodman's theory of symbols. These four questions must be discussed together since the interpretation of "refers to" in (C2) may have implications for the question of whether (1) states necessary and sufficient conditions, as well as for the question of whether (1) should be interpreted as a definition, which in turn depends on the question of which notions should be taken as the basic ones. Before we introduce and defend our position, we present an overview of possible answers, beginning with the question of how "exemplification" may be defined.

\subsection{Five accounts of exemplification}

Since denotation and exemplification are the two most important elementary forms of reference, one may opt for interpreting "refers to" in (C2) as either denotational or exemplificational reference. The first option corresponds to the following definition:

(D) $\mathrm{x}$ exemplifies $\mathrm{y}={ }_{\mathrm{df}} \mathrm{y}$ denotes $\mathrm{x} \wedge \mathrm{x}$ denotes $\mathrm{y}$.

The second option threatens to yield a circular definition: $\mathrm{x}$ exemplifies $\mathrm{y}$ $={ }_{\mathrm{df}} \mathrm{y}$ denotes $\mathrm{x} \wedge \mathrm{x}$ exemplifies $\mathrm{y}$. This can only be avoided if this option is understood as claiming that (1) should not be interpreted as defining "exemplification", but as introducing a basic notion. In short, it claims:

(E) "Exemplification" is a basic notion (and therefore undefined).

According to proposal (D), "reference" and "denotation" are basic notions of the theory of symbols; according to (E), "exemplification" is a third basic notion. Variants of both (D) and (E) could be generated by

${ }^{1}$ The secondary extensions of an expression $\mathrm{P}$ are the extensions of compounds featuring this expression, such as "P-description". For the explication of likeness of meaning in terms of secondary extensions see (PP, 221-238) and (Elgin 1983, 54-58); this approach was further developed by Heydrich (1993). 
reducing the basic notions to "denotation", or to "denotation" and "exemplification" respectively. For this purpose, "reference" would have to be defined disjunctively: $\mathrm{x}$ refers to $\mathrm{y}=_{\mathrm{df}} \mathrm{x}$ denotes $\mathrm{y} \vee \mathrm{x}$ exemplifies $\mathrm{y}$. The consequences of such a reduction will be discussed towards the end of this section.

A third proposal results if "refers to" in (C2) is interpreted as a basic form of reference, different from both denotation and exemplification. This implies that an additional term, say, "X-reference" has to be taken as basic:

(X) $\mathrm{x}$ exemplifies $\mathrm{y}==_{\mathrm{df}} \mathrm{y}$ denotes $\mathrm{x} \wedge \mathrm{x} X$-refers to $\mathrm{y}$.

If, however, one wants to stick as closely as possible to the original (1), it will be most natural to leave open the question which form of reference is meant by "refers to" in (C2). Thus, we get a fourth proposal:

(RD) $\mathrm{x}$ exemplifies $\mathrm{y}={ }_{\mathrm{df}} \mathrm{y}$ denotes $\mathrm{x} \wedge \mathrm{x}$ refers to $\mathrm{y}$.

Like (D), (RD) assumes that "reference" and "denotation" are basic notions. Nevertheless, the two proposals are not equivalent because "refers to" in (RD) may be denotational, but need not be so. ${ }^{2}$

A fifth proposal results if "reference" is assumed to be the only basic notion. This means that both "denotation" and "exemplification" have to be defined as unidirectional and bidirectional reference respectively. So, (R) comprises the following two definitions:

(R1) $\mathrm{x}$ denotes $\mathrm{y}={ }_{\mathrm{df}} \mathrm{x}$ refers to $\mathrm{y}$.

(R2) $\mathrm{x}$ exemplifies $\mathrm{y}=_{\mathrm{df}} \mathrm{y}$ refers to $\mathrm{x} \wedge \mathrm{x}$ refers to $\mathrm{y}$.

Of all these proposals, (X) may be dismissed straightaway. Firstly, it is implausible as an interpretation of Goodman since he never writes about such a third form of reference. This is confirmed by Elgin's explicit claim that there are exactly two basic forms of reference, denotation and exemplification (Elgin 1983, 5; 1993, 172; 1995, 66; 1997a, xvii; 1997b,

\footnotetext{
2 Again, a variant of (RD) could be introduced by defining "reference" disjunctively: $\mathrm{x}$ refers to $\mathrm{y}={ }_{\mathrm{df}} \mathrm{x}$ denotes $\mathrm{y} \vee \mathrm{x}$ exemplifies $\mathrm{y} \vee \mathrm{x} X$-refers to $\mathrm{y}$. This would turn (RD) into: $\mathrm{x}$ exemplifies $\mathrm{y}=_{\mathrm{df}} \mathrm{y}$ denotes $\mathrm{x} \wedge$ ( $\mathrm{x}$ denotes $\mathrm{y} \vee \mathrm{x}$ exemplifies $\mathrm{y}$ $\checkmark \mathrm{x}$ X-refers to $\mathrm{y}$ ). But this proposal is rather pointless. Instead of introducing "exemplification" as a disjunct in the second condition, one would better accept (E).
} 
101). Secondly, $(\mathrm{X})$ is not a sensible proposal. Instead of introducing a mysterious basic notion in order to define "exemplification", it would be better to introduce "exemplification" itself as basic. This leaves us with the four proposals (D), (E), (RD) and (R).

The differences between these proposals can be analysed with the help of some statements about reference, denotation and exemplification. To begin with, the following three should hold in any defensible account:

(2) $\mathrm{x}$ denotes $\mathrm{y} \rightarrow \mathrm{x}$ refers to $\mathrm{y}$.

(3) $x$ exemplifies $y \rightarrow x$ refers to $y$.

(4) $x$ exemplifies $y \rightarrow y$ denotes $x$.

In the context of Goodman's theory, these are uncontroversial statements. (2) and (3) express that denotation and exemplification are forms of reference; and (4) is just condition (C1) of (1). In all four proposals the three statements can either be proved as theorems or postulated without any problems. (All three are implied by (R1) and (R2), (RD) implies (3) and (4), and (D) implies only (4).) Consequently, there are no important differences between the four proposals with respect to the claims (2), (3) and (4).

But there are remarkable differences between the four proposals with respect to the following two statements, both of which must be rejected:

(6) $\mathrm{x}$ denotes $\mathrm{y} \wedge \mathrm{y}$ denotes $\mathrm{x} \rightarrow \mathrm{x}$ exemplifies $\mathrm{y}$.

As we will show, (5) and (6) have utterly implausible consequences and (5) cannot be defended as an interpretation of Goodman's writings. But there is no way to defend (D), (R) or (RD) without accepting (5) or (6), since (5) follows from (D) and from (R), and (6) is implied by all three proposals. $^{3}$ (E), however, implies neither (5) nor (6). Therefore, if one is not willing to accept (5) or (6), one has to defend (E), in other words, one has to accept "exemplification" as a basic notion. As far as we know, this has not been proposed unambiguously in the literature, although perhaps many writers have assumed it tacitly. We will defend it in this paper.

\footnotetext{
${ }^{3}$ In case of (RD), the uncontroversial postulate (2) is needed as well.
} 
Although (E) makes it possible to get rid of the unwanted theorems (5) and (6), this comes at a price. (1.1) cannot be accepted in its original form since (6) is implied by (1.1) and (2). Instead one may accept only a weaker reading of (1) such as:

(1.2) $\mathrm{x}$ exemplifies $\mathrm{y} \leftrightarrow \mathrm{y}$ denotes $\mathrm{x} \wedge \mathrm{x}$ exemplifies $\mathrm{y}$.

(1.3) $\mathrm{x}$ exemplifies $\mathrm{y} \rightarrow \mathrm{y}$ denotes $\mathrm{x} \wedge \mathrm{x}$ refers to $\mathrm{y}$.

Of course, these claims look suspicious. For (1.2) is equivalent to (4) and (1.3) is equivalent to the conjunction of (4) and (3). But if we adopt (E), we may interpret (1.2) as emphasizing once more that denotation in the opposite direction is only a necessary condition for exemplification. (1.3) provides an alternative, conditional instead of biconditional, reading of (1).

Such moves make sense only if one is going to defend (E). For the stronger claim (1.1) is implied by (R) and (RD); and in case of (D), nothing substantial can be gained by avoiding (1.1) since (6) follows from (D) directly (the same goes for $(\mathrm{R})$ as well). In short, there is no way to avoid (6) by replacing (1.1) with a weaker claim in any of the alternatives to (E).

\subsection{Introducing basic notions}

According to (E), "exemplification" is a basic notion of Goodman's theory of symbols. This raises the question of how exemplification may be introduced into his theory. For this purpose, a range of strategies can be used, preferably in combination. They can all be illustrated with passages from Elgin's With Reference to Reference. Firstly, examples may be used to point out the intended use of the predicate "x exemplifies $y$ ":

A tailor's swatch, for example, simultaneously exemplifies labels denoting fabric, pattern, colour, and weave (Elgin 1983, 79; our emphasis).

Thus, e.g., if disconnecting the respirator that keeps a patient alive is described as 'killing him', the action is brought to exemplify morally blameworthy labels. If it is described as 'ending his suffering', it is brought to exemplify morally praiseworthy labels (Elgin 1983, 90; our emphasis).

Secondly, one may refer to similar notions in ordinary language and show how they differ in meaning or usage: 
The samples we encounter are various, and the uses to which they are put diverse. The model home on a development site, the prototype of a jet plane, and the free bottle of shampoo which arrives in the mail are integral parts of sales campaigns. A sample problem worked out in a text is an illustration of characteristic problems and acceptable modes of solution in a given discipline. And an example of the way you can expect to be treated or the sort of person you are likely to become, may serve as a promise or threat (Elgin 1983, 71; our emphasis; cf. $W W, 65$ ).

But Elgin is not concerned with cataloguing the different roles that samples play. Rather, she wants to determine just what it is for something to function as a sample. This is completed by "Something serves as a sample when it functions as a symbol for a label it instantiates." (Elgin 1983, 72), which in this context indicates that "is a sample of" may be used in a much more general sense than in ordinary discourse. Thirdly, possible misunderstandings can be addressed:

Let us begin by considering a familiar sample - a chip of paint on a manufacturer's sample card. This particular chip is blue. [...] Under its standard interpretation, it is a sample of 'blue', [...] Its being a sample of 'blue' does not depend on its instantiation of the label being particularly conspicuous or striking as compared with its instantiation of other labels (Elgin 1983, 71; our emphasis).

Finally, one may discuss the relation of the newly introduced term to other technical terms that are already in use:

Exemplification then is like denotation in being a mode of reference, but it differs from denotation in direction. [...] Exemplification is not, however, the converse of denotation. To denote an object, a term need only refer to it. But to exemplify a term, an object must both refer to and instantiate that term (Elgin 1983, 73).

All in all, this procedure of introducing a basic term corresponds to the classic method of explication. According to Carnap (1971, §§2-3), explicating a term means replacing a pretheoretical term ("explicandum") by another term ("explicatum") that is more exact and embedded in a theory. As a preliminary step, the relevant use of the explicandum has to be identified and distinguished from other uses of the same term. The explicatum is required to meet four conditions. (i) In important cases, it can be used instead of the explicandum ("similarity"). (ii) Its use in a system of terms is laid down as precisely as possible. This can but need not be done 
by introducing an explicit definition. Finally, the explicatum needs to be (iii) as fruitful and (iv) simple as possible. All these points can be identified in the examples presented above. The first two groups of quotations show how "exemplifies", "is a sample of" and some further expressions are identified as explicanda and how the relevant uses of these terms are explained and illustrated by examples. The predicate " $\mathrm{x}$ exemplifies y" is then introduced as explicatum that can be used in place of the explicanda. Its use is regulated by statements such as (1) or the passage quoted last, which put forward the two conditions (C1) ("x exemplifies $y$ $\rightarrow \mathrm{y}$ denotes $\mathrm{x}$ ") and (C2) ("x exemplifies $\mathrm{y} \rightarrow \mathrm{x}$ refers to $\mathrm{y}$ "). These conditions ensure that the use of the explicatum is more exactly regulated than the use of the explicanda, even though the explicatum is not defined explicitly, but introduced as a basic notion. A more comprehensive look at Goodman's theory of symbols also shows that the explicatum is extraordinarily fruitful. The notion of exemplification plays a crucial role in Goodman's systematic accounts of artistic expression $(L A, 85-95)$ and of the symbolic functions of, for example, fictional labels $(L A, 21-26 ; 66)$, metaphors ( $L A$ 68-85) and abstract paintings ( $W W, 63-66)$; furthermore, as Elgin (1983, 81-93; 1996, 171-186) has shown, this explication also serves as a basis for analysing important parallels and contrasts between those symbolic functions and the role samples play in the sciences. Finally, the two conditions (C1) and (C2) give a simple account of the core features of "exemplification".

Nevertheless, one may feel inclined to object that introducing "exemplification" as a basic term leaves this notion unexplained and consequently turns "exemplification" into an unintelligible and useless technical term. But if such objections were justified, they would be similarly valid against denotation - or any other basic notion for that matter. After all, no theory can be developed without relying on one or more undefined notions. Still, one may want to insist on admitting as basic no technical terms but only notions from ordinary language (cf. Textor 2008, 349). But this strategy would result in giving up the core advantages of Goodman's theory. For the ordinary notions of exemplification and sample are far less fruitful. They are, for example, quite useless for explaining how metaphors and fictional labels function. 
There is one marked difference between Goodman's introduction of exemplification in Languages of Art and the one given by Elgin. Goodman starts with the example of a tailor's swatch and then proceeds to introduce the following conditions $(L A, 53)$ :

(7) There are two conditions for $x$ exemplifying the property $y:$ (C3) $\mathrm{x}$ possesses $\mathrm{y}$ and $(\mathrm{C} 2) \mathrm{x}$ refers to $\mathrm{y}$.

A carmine swatch exemplifies carmine because it possesses this colour and refers to it. If one starts with such an example, it is more natural to explain exemplification in terms of properties and possession rather than labels and denotation. But right after the passage just reported, Goodman argues that exemplification should be explained by appeal to labels and denotation as in (1). There is an obvious reason for this move. Goodman's nominalism does not allow for properties but explains possession of properties in terms of being denoted by labels ( $L A, 54-57$; cf. $M M$, 59, note 4). Yet, there is another reason not mentioned by Goodman. (1) introduces "exemplification" using the semiotic terms "label", "denotation" and "reference", whereas (7) relies on "properties" and "possession (of properties)", which are not semiotic terms. Therefore, choosing the latter alternative would place non-semiotic terms at the heart of the theory of symbols, as well as introduce an ontological commitment to properties. All this is avoided by (1). Nonetheless, Goodman's strategy of replacing properties by labels is often challenged by pointing out that there are apparently not enough labels (for some properties do not have a label) as well as too many labels (for some different labels refer to the same properties). Goodman (LA, 54-57; 67) and Elgin (1983, 76-78) have addressed both problems but the details of this issue are quite irrelevant to the topic of this paper. We therefore restrict the following discussion to Goodman's official theory, which explains exemplification in terms of labels, denotation and reference.

As we have introduced (E), all three notions, "denotation", "exemplification" and "reference", are taken to be basic, and hence undefined. But as mentioned before, "reference" could easily be defined as the disjunction of its basic forms. However, such a definition would presuppose a complete list of the basic forms of reference and it is not clear 
that such a list can be given once and for all. In one passage, for example, Goodman points out that reference cannot always be interpreted as denotation or exemplification, since an object may be used as a symbol for something on the basis of almost any relation $(L A, 65)$. While this rules out defining "reference" by enumerating all its basic forms, it leaves open the possibility of introducing "reference" using an open list of such forms. On the other hand, treating "reference" as a third basic notion also has its drawbacks. The resulting symbol theory will have more basic notions and consequently be less simple (in the sense explained in The Structure of Appearance), systematic and coherent (cf. SA, III; PP, VII). Nevertheless, we think it is better on the whole to accept these disadvantages than to rely on the rather dubious assumption that denotation and exemplification can be identified as the only basic forms of reference once and for all. For this reason, we stick to the original version of (E) that includes "reference" as a basic notion.

\section{Goodman and Elgin on exemplification}

In this section we investigate whether Goodman's and Elgin's writings support our interpretation (E) of (1) that treats "exemplification" as a basic notion of Goodman's symbol theory. Since at least Goodman never explicitly formulates (E) we have to proceed indirectly and ask first whether there is an official definition of "exemplification" in Goodman's writings.

\subsection{Is there an official definition of "exemplification"?}

In all of Goodman's and Elgin's writings there is but one place with an explicit hint that "exemplification" might be defined. The index of Languages of Art contains an entry "exemplification, definition of" referring to page 52. Presumably the statement in question is:

An object that is literally or metaphorically denoted by a predicate, and refers to that predicate or the corresponding property, may be said to exemplify that predicate or property $(L A, 52)$.

However, it is quite questionable whether this hint can be taken seriously. Should this passage be interpreted as providing a definition in a strict 
sense? Even Goodman's official definition of "exemplification"? A positive answer is implausible for several reasons. Firstly, Goodman did not write the Index to Languages of Art (cf. LA, vi). Secondly, the structure of Goodman's text speaks against such an interpretation. The quoted passage is nowhere referred to as a definition in the text itself, it occurs before the chapter heading Exemplification and there are many more or less similar passages which could be interpreted as definitions of "exemplification" with equal (im)plausibility. Thirdly, an entry “..., definition of" in the index does not imply that the page it refers to contains a definition of "...", for such a definition may only be talked about. There is, for example, an entry "expression, definition of" referring to a passage $(L A, 95)$ where Goodman explicitly states that he is not defining "expression". Finally, it is implausible that Goodman should include the term "property" in an official definition, since he uses it only to conform to customary ways of speaking.

\subsection{Can "exemplification" be defined?}

All relevant alternatives to our proposal (E) are suggestions for defining "exemplification". In order to judge their plausibility from an exegetic point of view, we discuss passages that throw some light on the question of whether and how "exemplification" could be defined. At least for (RD) there are a number of formulations in Goodman's writings that could, with some plausibility, be read as definitions of "exemplification", namely those stating that exemplification is being denoted (or possession) plus reference (e.g. LA, 53). If "is" here is read in a definitional sense, (RD) results directly. But let us first turn to proposal (D).

According to (D), the relation called "reference" in (C2) is denotation. But this view is challenged by passages in Of Mind and Other Matters where Goodman explicitly claims that exemplification is not a denotational form of reference. For example:

An extremely important but often overlooked form of nondenotational reference is exemplification [...] Exemplification, then, far from being a variety of denotation, runs in the opposite direction, [...] Exemplification indeed involves denotation, by inversion, yet cannot be equated with the converse of denotation ( $M M, 59$; Goodman's emphasis) 
Although exemplification is not a denotational relation at all, $[\ldots](M M, 60)$

A notably clear statement can be found in Goodman's reply to Richard Martin's complaint that aesthetic relations, such as exemplification, should not be subsumed under reference or denotation (Martin 1981, 261):

I do not, as Martin implies in one place, "subsume [these] relations under denotation" but rather under the broader relation of reference. Exemplification and expression are nondenotational but referential relations ( $M M, 92$, note 27; Goodman's emphasis).

This is Goodman's interpretation of his position in Ways of Worldmaking. His formulations in Languages of Art are somewhat less unambiguous. However, Goodman writes in a letter to Monroe Beardsley:

[...] presenting for apprehension is something more than merely having - it is emphasizing, calling attention to, showing forth, exemplifying (and so referring to though not denoting) the properties in question. I use "reference" for the general relation of symbolization; exemplification and denotation are different kinds of reference (see, e.g., Languages of Art, pp. 51-52, 57ff, 92) (Goodman 1975, 25; his emphasis).

This leaves no doubt that Goodman considers the claim that exemplification is a nondenotational relation to be part of the position he presented in Languages of Art. Hence, Goodman explicitly rejects (D) as an interpretation of Languages of Art. This, however, leaves open the question of whether denotation might be a necessary or a sufficient condition for the relation of reference mentioned in (C2). We will address this issue a bit further on. For now, we may note that the passages discussed so far imply that Goodman accepts a second form of reference that cannot be reduced to denotation. Interpretation (E) assumes that this nondenotational form of reference simply is exemplification, which consequently needs to be accepted as a basic notion. We will therefore briefly look at what Goodman and Elgin write about the basic notions of their theory of symbols, extending the discussion to include proposals (R) and (RD).

In Routes of Reference and Reconceptions Goodman comments on the conceptional structure of his theory of symbols: 
"Reference" as I use it is a very general and primitive term, covering all sorts of symbolization, all cases of standing for. As a primitive relation, reference will not be defined but rather explicated by distinguishing and comparing its several forms ( $M M, 55$; Goodman's emphasis).

The basic notion is reference or symbolization, the relation between a symbol and whatever it stands for in any way. As primitive, this relation is not defined but explicated in terms of its several varieties, among them (1) denotation, reference by a word or other label to something it applies to, as in naming or predication, and (2) exemplification, reference by an instance, as a sample, to a label denoting it $(R, 124$; Goodman's emphasis).

These passages leave no doubt that reference is a basic notion of Goodman's theory of symbols, and hence undefined. With respect to the various forms of reference, Goodman also makes clear that he does not intend to define but only to distinguish and compare these notions. We should therefore not expect definitions of "denotation" or "exemplification". Neither should we assume that Goodman is committed to the claim that these notions could be defined with reference to "reference" (and logical notions) alone - or in any other way.

In other respects, however, these passages pose some problems of interpretation. How should one understand Goodman's announcement that he will explicate the notion of reference? Why is only reference called "basic" and "primitive"? And what is meant by these attributes?4 We start with Goodman's use of "explication". Firstly, explications are often thought of as explicit definitions satisfying certain criteria. But this cannot be what Goodman wants to express since he does not intend to define "reference". Secondly, it is implausible that Goodman intends to give an explication in Carnap's classical sense. Although this would not necessarily call for an explicit definition, it would imply that Goodman intends to give a characterization of the notion of reference meeting Carnap's criteria of adequacy (cf. section 1.3). But Goodman adopts only

4 In Routes of Reference, exemplification is also called an "elementary" form of reference. But, as the headings of the main sections in Routes of Reference suggest, this probably means only that exemplification is a non-complex form of reference; that is, no chain of reference (cf. $M M, 61 \mathrm{f} ; 70$ ). 
the less ambitious goal of distinguishing and comparing forms of reference. It may therefore be best to interpret him in a third way as using "explication" not terminologically but only in the sense of "clarifying a notion", corresponding to the first step of Carnap's method of explication. ${ }^{5}$ This interpretation could be supported by interpreting "basic" and "primitive" in the way they are used in The Structure of Appearance (4546). This would imply that "reference" is introduced without being defined. On the other hand, the basic and primitive notions in The Structure of Appearance are introduced as a basis for defining further notions. But, as we have already seen, this is not what Goodman declares as his intention in the quoted passages. He does not claim to define "denotation" or "exemplification", much less to develop a system of semiotic notions using the constructional techniques of The Structure of Appearance. Consequently, it is not clear whether the quoted passages can sensibly be interpreted with reference to Goodman's theory of constructional systems in The Structure of Appearance. All in all, it seems fairly questionable whether the fact that Goodman calls only reference "basic" and "primitive" should be taken to imply that "denotation" and "exemplification" are notions that are not basic but defined. The best interpretation may be that Goodman leaves open the question of whether and how "denotation" and "exemplification" can be defined in a strict sense. This respects at least the general line of The Structure of Appearance, which calls for selecting the primitive notions according to criteria that can be assessed only on the basis of a constructional system (e.g. simplicity). To sum up, Goodman does not claim to give a definition of "exemplification", but only to distinguish and compare exemplification and other forms of reference. This neither excludes nor implies that "exemplification" could be defined. It therefore seems sensible to treat

\footnotetext{
5 In Goodman's writings, "explication" and "explicative" appear only casually and with various meanings. In the context of The Structure of Appearance explications with explicit definitions are called "definitions" $(S A, \mathrm{I})$ or "(explicative) definitions" $(P P, 4)$; informal explanations of notions are called "informal explications" $(S A, 19$, note 8). In Fact, Fiction, Forecast (65, note 2), his use of "explication" can be interpreted in all three ways we have distinguished.
} 
exemplification as an undefined notion (at least at the present stage of development of Goodman's theory). This interpretation can be supported by Goodman's passages that introduce the notion of exemplification (e.g. $L A, 51 \mathrm{ff}$ ). There we find all the strategies for introducing an undefined notion that we have discussed in section 1.3, although Goodman is somewhat less strict, especially in his liberal use of property-talk.

Elgin's writings, however, are unambiguous:

Goodman $[\ldots]$ recognizes two primitive semantic relations - denotation and exemplification - and constructs a variety of complex modes of reference out of them (Elgin 1997a, xvii; 1997b, 101).

Like Goodman, I recognize two basic modes of reference - denotation and exemplification (Elgin 1995, 66).

According to Elgin, denotation and exemplification are the two basic and primitive forms of reference, and this is also her interpretation of Goodman. We read these quotes as claiming that "denotation" as well as "exemplification" are basic notions of the theory of symbols. Even without explicitly using the word "undefined", treating "denotation" and "exemplification" as notions which are on the same conceptual level not only implies that "exemplification" may not be defined as a form of denotation but also rules out that "exemplification" is defined any more than "denotation". But all interpreters we know of agree that Goodman and Elgin do not define "denotation", and there is not a single passage that could plausibly be read as such a definition (cf. $M M, 55-59$; LA, 3-6; Elgin 1983, 19). 6

This point can also be made more generally. The whole discussion about basic, primitive and undefined notions could be repeated for "denotation" instead of "exemplification". The only difference would be that there is not even a suspicious entry in the index of Languages of Art. Consequently, it is hard to see why one should believe that Goodman intended to treat the

\footnotetext{
${ }^{6}$ One could venture the claim that Goodman defines " $x$ denotes $y$ " as "x applies to $y$ " (in analogy to Quine's alleged definition as " $\mathrm{x}$ is true of $\mathrm{y}$ ", which would be too narrow for Goodman). But this is not a definition in any interesting sense but only the introduction of an alternative label for the same relation.
} 
two notions "denotation" and "exemplification" markedly different with respect to their introduction and definition. To put it more polemically, whoever thinks that Goodman has or needs a definition of "exemplification" should also tell us where to find a definition of "denotation" or at least why we should not need it. It is much more plausible to assume that both terms are not defined but treated as basic in Goodman's theory.

All in all, the points discussed so far undermine interpretations (D), (R) and also (RD) since these proposals all assume that Goodman defines "exemplification"; 7 and they speak in favour of (E) since (D), (R) and (RD) are all the relevant alternatives to $(\mathrm{E})$. To confirm this result, we turn to the question of whether denotation in the same direction might be a necessary or sufficient condition for exemplification.

\subsection{The relationship between denotation and exemplification}

In his letter to Beardsley, Goodman explicitly rejects the idea that denotation in the same direction is necessary for exemplification: "referring to though not denoting" (Goodman, 1975, 25). Further support for this interpretation of Languages of Art is provided by the text itself. Firstly, there is not a single passage suggesting that the labels a sample can exemplify should be restricted to those it denotes. Rather, Goodman introduces exemplification with examples that make it utterly implausible to think that the samples denote the labels they exemplify. Even though they exemplify, a tailor's swatches in general do not denote at all, and Goodman does not claim that they do, but only that they refer to the labels they exemplify. Since denotation has not only been introduced before exemplification, but is also used in the explanation of exemplification, Goodman surely would have explicitly pointed out that denotation in the same direction were necessary for exemplification if this indeed were true.

\footnotetext{
${ }^{7}$ An additional problem challenging $(\mathrm{R})$ is Goodman's remark "denotation implies reference between two elements in one direction while exemplification implies reference between the two in both directions" ( $L A, 59)$. According to $(\mathrm{R})$, we should find "is" in place of "implies". If (R) were correct, Goodman had no reason to prefer the weaker claim to the stronger.
} 
Secondly, Goodman draws attention to the fact that usually the practical distinction between instances of denotation and exemplification is easily made in the case of ordinary language:

In ordinary language, the reference of "man" to Churchill, and of "word" to "man", is unequivocally denotation; while if Churchill symbolizes "man", and "man" symbolizes "word", the reference is unequivocally exemplification $(L A, 57-58)$.

Writing that the reference from Churchill to "man" is unequivocally exemplification would be highly misleading if this reference were also an instance of denotation. If denotation in the same direction were necessary for the reference mentioned in (C2), there would be an ambiguity in every instance of exemplification; for a sample then could not exemplify a label without denoting it as well.

Finally, Goodman's extensive discussion of a difference in direction between denotation and exemplification (cf. $L A, 52,57 \mathrm{ff}, 65 ; M M, 59,82$; $R, 36$ ) would completely miss the point if denotation in the same direction were necessary for exemplification. If this were true, the crucial difference between denotation and exemplification would have nothing to do with a difference in direction, but would amount to exemplification being bidirectional denotation while "normal" denotation would be just unidirectional denotation, as claimed in proposal $(\mathrm{R})$.

In short, denotation in the same direction is not a necessary condition for exemplification and, hence, statement (5) - " $\mathrm{x}$ exemplifies $\mathrm{y} \rightarrow \mathrm{x}$ denotes y" - is false. As a consequence, neither (D) nor (R) can be defended as an interpretation of Goodman's position, since they both imply (5).

However, whether denotation is sufficient for the relation of reference in (C2) is a more difficult question. Goodman addresses it when he deals with the special case of self-reference $(L A, 59)$. After mentioning some examples he notes four "theorems":

(a) If $x$ exemplifies $y$, then $y$ denotes $x$. [= (4)]

(b) $\mathrm{x}$ and $\mathrm{y}$ denote each other if and only if they exemplify each other.

(c) $x$ exemplifies $x$ if and only if $x$ denotes $x$.

(d) If $\mathrm{x}$ exemplifies and is coextensive with $\mathrm{y}$, then $\mathrm{x}$ denotes and exemplifies $\mathrm{x}$ ( $L A, 59$, note 9$)$. 
The crucial claim is (b) since (a) is uncontroversial, (c) follows from (b), and (d) from (a) and (b). ${ }^{8}$ That Goodman calls (b) a "theorem" suggests that he thinks it can be derived from some more fundamental statements about denotation and exemplification. But the uncontroversial (a) only implies "half" of (b): "If $x$ and $y$ exemplify each other, then they denote each other". What is missing for deriving (b) is the already mentioned claim:

(6) $\mathrm{x}$ denotes $\mathrm{y} \wedge \mathrm{y}$ denotes $\mathrm{x} \rightarrow \mathrm{x}$ exemplifies $\mathrm{y}$.

In (D), (R) and (RD), (6) can be derived from the respective definitions of "exemplification" (together with, in case of (RD), the uncontroversial (2)). In interpretation (E), however, (6) cannot be derived as a theorem unless one postulates (1.1) or some other suitable statement. Thus, from an exegetic point of view, the fact that Goodman's theorems (b)-(d) presuppose (6) supports (D), (R) and (RD), since (6) is a theorem in those interpretations, but an independent claim in (E). From a systematic point of view, however, the opposite is true. Unlike (a), (6) is highly problematic, as will be shown in section 3 . This will amount to a powerful argument for (E): in (E), (6) can be given up, but not in (D), (R) and (RD).

Before picking up this argument, we may note that, again, the writings of Elgin fit our proposal (E) even better. Elgin formulates neither the theorems mentioned nor (1.1) which, together with the uncontroversial postulate (2), implies the problematic claim (6). Instead of (1.1) she uses the unproblematic (1.3), which is compatible with (E), as the "official" characterization of exemplification: "When an object exemplifies a label, it both refers to and instantiates that label" (Elgin 1983, 73).

\section{The debate on the notion of exemplification}

We now turn to systematic arguments in favour of (E), analysing the debate on the notion of exemplification. Since (E), (D), (R) and (RD) are

${ }^{8}$ The plausibility of (d) depends on how exemplification of coextensive labels is dealt with. Goodman considers some alternative approaches in $L A(54-56)$. We will not discuss this issue. 
all proposals for interpreting the two conditions for exemplification that Goodman puts forward in (1), we will focus on the question of how "refers to" has to be understood in $(\mathrm{C} 2)$. We will not discuss positions that cannot claim to be at least broadly Goodmanian. This includes not only the suggestion that the notion of exemplification should be abandoned, but also all non-semiotic accounts of exemplification; that is, all proposals that replace "refers to" in condition $(\mathrm{C} 2)$ with a non-semiotic relation. ${ }^{9}$ Some authors advocate such a move since they think that Goodman's account of exemplification faces insurmountable problems. We attempt to show that these problems vanish if interpretation (E) is accepted.

\subsection{Dempster's dilemma}

Douglas Dempster published a widely read critique of Goodman's notion of exemplification. He considers three possible readings of "refers to" in condition (C2) of (1) (Dempster 1989, 407-410):

(8) $x$ exemplifies $y$.

(9) $\mathrm{x}$ denotes $\mathrm{y}$.

(10) $x$ refers to $y$ in some other way.

Option (10) leads to definition (X). It has already been criticized in section 1 and Dempster rejects it as well. Since Dempster argues that the two remaining proposals lead to unsolvable problems, his attack may be reconstructed as a dilemma.

The first horn of the dilemma is generated by (8), if "exemplification" is defined as

(11) $\mathrm{x}$ exemplifies $\mathrm{y}={ }_{\mathrm{df}} \mathrm{y}$ denotes $\mathrm{x} \wedge \mathrm{x}$ exemplifies $\mathrm{y}$.

Since this is obviously a circular definition, no author has seriously considered adopting it (cf. Dempster 1989, 407; Steinbrenner 2005, 230).

${ }^{9}$ For non-semiotic accounts of exemplification see, e.g., Beardsley 1975, 13-19; 1978, 102-106; Dempster 1989, 410-412; Textor 2008, 351-359. Beardsley sometimes also shows a tendency to give up the notion of exemplification altogether (cf. 1978, 106). 
The second horn is the result of choosing option (9); that is, of adopting definition (D) of "exemplification":

(D) $\mathrm{x}$ exemplifies $\mathrm{y}=_{\mathrm{df}} \mathrm{y}$ denotes $\mathrm{x} \wedge \mathrm{x}$ denotes $\mathrm{y}$.

Dempster $(1989,407)$ argues that (D) provides the only acceptable interpretation of Goodman's position since (11) has to be rejected for the obvious reason already mentioned. Probably, this interpretation has also been inspired by Goodman's theorems of self-reference. But we may note that although the theorems follow from (D), one can accept them without being committed to (D). As we have discussed in section 2, denotation could be sufficient but not necessary for the relation of reference that is called for in (C2). ${ }^{10}$ Nevertheless, proposal (D) is materially inadequate for three reasons (Dempster 1989, 407f).

Firstly, (D) blurs the distinction between exemplification and denotation, and consequently between samples and labels. The reason is that according to (D) there are no longer two fundamental forms of reference but only one, denotation, which can be unidirectional or bidirectional. Exemplification then simply is bidirectional denotation. But this contradicts not only Goodman's and Elgin's frequent assurance that there are two fundamental forms of reference, but also their insisting on a difference in direction. For if exemplification is denotation in both directions, there is no "opposite" direction left that could distinguish exemplification from denotation. ${ }^{11}$ Furthermore, labels would no longer be distinguished from samples as being denotational symbols because samples would denote as well.

${ }^{10}$ This may be what Dempster wants to express when he writes: "This [(D)] may be too strong. Goodman seems to hold only that compound denotation is sufficient for exemplification, indeed sufficient for compound exemplification" (1989, 407, note 24). Dempster's use of "compound" is ill-advised since it suggests a chain of reference, but exemplification is not a complex form of reference. We will use therefore "bidirectional" instead of "compound".

${ }^{11}$ The difference in direction could be saved if (D) were interpreted as requiring not bidirectional denotation, but only denotation that presupposes denotation in the opposite direction. But then it would be impossible to say which of the two relations is exemplificational. This is the objection of symmetry, which is discussed next. 
Secondly, (D) renders exemplification symmetric. If the conditions for $\mathrm{x}$ exemplifying y are met, $\mathrm{y}$ exemplifies $\mathrm{x}$ as well. But surely, a sample is not exemplified by everything it exemplifies. That a colour sample exemplifies "red" does not imply that "red" exemplifies that colour sample. Even restricted to cases of bidirectional denotation, exemplification is not symmetric. If "word I have not thought of yesterday at 3:07 a.m." is used as a sample for a predicate and hence exemplifies "predicate", then this does not imply that "predicate" exemplifies "word I have not thought of yesterday at 3:07 a.m.", although the two expressions denote each other.

Thirdly, according to (D), bidirectional denotation is sufficient for exemplification since (D) implies (6). But there are countless counterexamples featuring bidirectional denotation without exemplification. On the one hand, there are cases of bidirectional denotation as in the preceding example. On the other hand, there are cases of self-denotation, which perhaps are even more convincing counter-examples. Here are two:12 When asked to illustrate what counts as a German word, I may start with "kurz". This word denotes itself, although in the context mentioned it does not exemplify itself, but rather "German word". Or suppose I am writing in blue ink on a postcard "The blue sea is here and I wish you were beautiful". The word-token "blue" denotes itself without exemplifying itself, for it does not exemplify at all. ${ }^{13}$

Constructing a dilemma with the two horns (11) and (D), as Dempster does, presupposes that "exemplification" should be defined. In a nutshell, the dilemma is this: in Goodman's definition of "exemplification" (x exemplifies $\mathrm{y}={ }_{\mathrm{df}} \mathrm{y}$ denotes $\mathrm{x} \wedge \mathrm{x}$ refers to $\mathrm{y}$ ) "reference" must either be

12 Dempster's counter-examples are of limited use $(1989,408)$ since he does not specify any context of use. Therefore, one may imagine circumstances in which the label in question would indeed be used to exemplify as well. Similar counter-examples can be found in Textor $(2008,349 f)$.

13 The problem is not that we sometimes do not know what a symbol exemplifies. This indeed happens if we face difficulties in finding out about the symbol system in use or in making it explicit; this difficulty affects denotational systems as well, but it is more common with exemplificational systems because they are less standardized. The relevant problem is that according to (D) a symbol would sometimes have to exemplify something we know it does not exemplify in the given context. 
interpreted as exemplificational or as denotational reference; but in the first case the definition is circular, in the second it is inadequate; hence Goodman's definition of "exemplification" has to be rejected.

The dilemma vanishes if one gives up the presupposition that "exemplification" should be defined. This opens the possibility of interpreting "refers to" in (C2) as exemplificational without running into an inacceptable circularity. Goodman's statements expressing (1) may be read not as defining a term but as introducing a basic notion. Since "refers to" in (C2) now is exemplification, it can no longer be denotation. This does not imply that an exemplificational relation cannot be accompanied by a denotational relation in the same direction. But even if there is such a denotational relation it is different from the exemplificational one. Consequently, the problems of the second horn disappear. Firstly, it is guaranteed that the difference between exemplification and denotation as well as between samples and labels remains clear. There are two basic forms of reference, which differ in direction. Of course, it can happen that we run into problems when we try to decide whether a particular symbol denotes or exemplifies what it refers to. But the epistemological question of what criteria may be used for finding out about whether something denotes or exemplifies must be distinguished from the question of what the conditions are for something to denote or exemplify something. In this paper we exclusively deal with such conditions, but not with epistemological criteria. Secondly, exemplification is not forced to be symmetrical if bidirectional denotation is not sufficient for exemplification. Even in cases of bidirectional denotation, there are still four possibilities: no exemplification, exemplification in one or in the other direction only, or exemplification in both directions. Thirdly, the counterexamples can easily be dealt with since bidirectional denotation and selfdenotation are no longer sufficient for bidirectional exemplification and self-exemplification respectively. Even though the expressions "word I have not thought of yesterday at 3:07 a.m." and "predicate" denote each other, there can now be situations where the second does not exemplify the first one; and although "kurz" and "blue" written in blue ink denote themselves, they need not exemplify themselves. For example, they do not exemplify themselves in the contexts described above. 
Incidentally, we may note that the problems discussed are independent of the definitional nature of (D). They also challenge the corresponding claim that bidirectional denotation is necessary and sufficient for exemplification. If we give up the idea of defining "exemplification", we can replace (11) by (1.2) and thereby avoid the charge of circularity. If, on the other hand, we replace (D) by

(12) $\mathrm{x}$ exemplifies $\mathrm{y} \leftrightarrow \mathrm{y}$ denotes $\mathrm{x} \wedge \mathrm{x}$ denotes $\mathrm{y}$,

we still face all three objections.

Dempster's dilemma could also be attacked in another way, by claiming that the alternatives he considers are not exhaustive. After all, there are more than three possibilities how "exemplification" could be defined. Specifically, there are (R) and (RD), which are quite obvious proposals, although Dempster manifestly has overlooked them completely. However, this strategy for dealing with Dempster's dilemma ultimately fails: (R) and (RD) are materially inadequate as well.

\subsection{Extending the argument}

No new objections are needed for attacking (R) and (RD). According to $(\mathrm{R})$, "reference" is the only basic notion and "denotation" as well as "exemplification" are defined in terms of reference:

(R1) $\mathrm{x}$ denotes $\mathrm{y}={ }_{\mathrm{df}} \mathrm{x}$ refers to $\mathrm{y}$.

(R2) $\mathrm{x}$ exemplifies $\mathrm{y}={ }_{\mathrm{df}} \mathrm{y}$ refers to $\mathrm{x} \wedge \mathrm{x}$ refers to $\mathrm{y}$.

This proposal has the obvious advantage that it manages to do with one basic notion only. But it has to be rejected for the same reasons as (D). Firstly, it obscures the distinction between exemplification and denotation and hence between samples and labels. Suggesting that there is only one basic form of reference and that exemplification is bidirectional reference is neither compatible with claiming that there are two fundamentally distinct forms of reference, nor with a difference in direction between denotation and exemplification. Secondly, (R) turns exemplification into a symmetric relationship, which is implausible as we have seen already. Thirdly, the counter-examples challenging (D) cause the same troubles for advocates of $(\mathrm{R})$. They show that bidirectional denotation is not sufficient 
for exemplification, but (R1) and (R2) imply exactly the contrary, namely (6).

Proposal (RD) accepts two notions as basic and defines

(RD) $\mathrm{x}$ exemplifies $\mathrm{y}={ }_{\mathrm{df}} \mathrm{y}$ denotes $\mathrm{x} \wedge \mathrm{x}$ refers to $\mathrm{y}$.

Although this has some plausibility as an interpretation of Goodman's text (cf. section 2.2), it nonetheless has to be rejected since it cannot deal with the counter-examples any more than (D) and (R). The reason is that (6) follows from (RD) and the uncontroversial (2) (" $\mathrm{x}$ exemplifies $\mathrm{y} \rightarrow \mathrm{x}$ refers to $\mathrm{y}$ "). Bidirectional denotation therefore is sufficient for exemplification according to (RD) and this is all that is needed for setting up the counter-examples. Again, the problems challenging (R) and (RD) cannot be evaded by giving up the definitions (R) and (RD) and resorting to the corresponding biconditional claims.

To sum up, (D), (R) and (RD), the alternatives to our proposal (E), are inadequate since (6), which is responsible for the counter-examples, cannot be given up in those accounts. Furthermore, although (1.1) could be held together with (E), this makes little sense since (1.1) and (2) together imply (6). Consequently, if (E) is adopted, (1.1) must be replaced by the weaker (1.2) or (1.3). This move is possible only because (E) does not imply (1.1) as (R) and (RD) do. Albeit (1.1) could also be given up in (D), this would be of little help, since (D) directly implies (6); consequently the unwanted implications cannot be avoided by replacing (1.1) with (1.2) or (1.3).

\subsection{Self-reference}

Importantly, the counter-examples discussed provide good reasons for rejecting not only proposals (D), (R) and (RD) but Goodman's theorems of self-reference as well. The counter-examples directly attack (b) and (c), which consequently must be given up. Once more, this can be done only in (E), since (b) and (c) are not theorems in a strict sense in (E). (D), (R) and (RD), on the other hand, imply (6),14 which together with the uncontroversial (a) implies the theorems (b)-(d). ${ }^{15}$

${ }^{14}$ In case of (RD), (2) is needed as well.

15 (d) or its negation can be added to the postulates of (E), which are (2), (3), (4), 
In (E) the problems are solved by accepting only (a), whereas (b) and (c) have to be replaced by weaker claims, which follow from (1.2) as well as from (1.3):

(13) If $x$ and $y$ exemplify each other then they denote each other.

(14) If $x$ exemplifies $x$ then $x$ denotes $x$.

This completes our argument in favour of (E) and against (D), (R) and (RD). In the rest of this section we briefly look at two interpretations of (1) we have not discussed so far.

\subsection{Peltz's account}

Richard Peltz has presented a further interpretation of "refers to" in (1). He complains that Goodman characterized the reference mentioned in (C2) only negatively as non-denotational and consequently did not make clear that it is indeed a form of reference. As a remedy, he suggests replacing reference with denotation in (C2), but in a different way than (D) or (12) do. His proposal can be reconstructed as follows: 16

(15) $\mathrm{x}$ exemplifies $\mathrm{y} \leftrightarrow \mathrm{y}$ denotes $\mathrm{x} \wedge \mathrm{x}$ denotes everything denoted by $\mathrm{y} .17$

This proposal is meant not as an interpretation but as a modification of Goodman's account; Peltz does not claim to explain what Goodman wrote or meant, but what he should have written. According to (15) the red colour-sample exemplifies "red" if and only if "red" denotes the sample and the sample denotes everything red. Peltz's move is to replace the condition that $\mathrm{x}$ must refer to $\mathrm{y}$ by the condition that $\mathrm{x}$ must denote everything denoted by $y$, which includes $x$ itself. Now, Goodman himself and (1.2) or (1.3); (6) still does not follow.

${ }^{16}$ The reason why Peltz favours (15) may be related to his view that denotation is the paradigmatic form of reference. He mistakenly attributes this stance to Goodman: "For Goodman the paradigm of symbolism is denotation. To be a sign or symbol is to denote" (Peltz 1972, 73).

${ }^{17}$ Peltz writes (1972, 81): “'A exemplifies B' can mean “'B' denotes A (A possesses B) and 'A' denotes what 'B' denotes"'. This formulation raises some further problems, which are discussed by Dempster $(1989,401-403)$ and Coldron $(1982,90$ 93). Additionally, Peltz would need to understand (15) as a definition given his motivation for introducing such a claim. 
notes that a sample which lacks an established denotation sometimes can be understood as denoting everything that is denoted by the label it exemplifies; it then will function as a label that exemplifies itself $(L A, 63)$. But this is typically not possible for samples that already have an established denotation. Even if "long" is used as a sample for short words and consequently exemplifies "short", it cannot take over the denotation of "short" since it denotes long objects, not short ones. Peltz tries to avoid this problem by introducing a notion of semi-metaphorical exemplification that would allow for claiming that "long" metaphorically denotes the objects literally denoted by the exemplified label "short" (Peltz 1972, 84).

Apart from introducing the rather dubious notion of semi-metaphorical exemplification, Peltz's proposal fails for two reasons. Firstly, every symbol that denotes itself meets the conditions mentioned in (15) and consequently exemplifies itself. Hence we have the same counter-examples as with (D). Secondly, while Goodman requires that a sample refers to the label it exemplifies, Peltz requires that it denotes what the exemplified label denotes. This raises the problem of coextensive labels. If Socrates exemplifies "rational" and "rational" is coextensive to "risible", then Socrates exemplifies "risible" according to (15); but surely, Socrates can exemplify "rational" without exemplifying "risible" (cf. LA, 55; Elgin 1983, 76). 18

\subsection{Steinbrenner's account}

Steinbrenner has presented an interpretation of (1) which is designed to counter the charge of circularity. His proposal can be put as follows (Steinbrenner 1996, 89; 2005, 231):

(16) An object $\mathrm{x}$ exemplifies a label $\mathrm{y} \leftrightarrow \mathrm{x}$ belongs to the extension of $\mathrm{y} \wedge \mathrm{x}$ refers to $\mathrm{y} \wedge \mathrm{x}$ indirectly refers to the extension of $\mathrm{y}$.

${ }^{18}$ Dempster already raises these objections $(1989,403 \mathrm{f})$. But he claims that Peltz faces the problem of coextensive labels because he allegedly holds that the objects referred to by exemplification are extensions. This is a misinterpretation of Peltz, who shares Goodman's view that samples exemplify labels. Rather, the problem of coextensive labels is generated by the second condition in (15). 
Presumably, the third condition is to be understood as equivalent to " $\mathrm{x}$ indirectly refers to the objects belonging to the extension of $y$ " since he gives the following reference for this condition: "Exemplification relates the symbol to a label that denotes it, and hence to the things (including the symbol itself) in the range of the label" ( $L A, 92)$. Proposal (16) reformulates condition (C1) of (1) by replacing "is denoted by" with the equivalent "belongs to the extension of" and it adds a third condition that is trivially met and hence superfluous. But it gives no answer to the question of how "refers to" is to be understood in (C2) since (16) retains the original wording of condition (C2). Consequently, Steinbrenner's proposal provides no solution to the problem of circularity that results if "refers to" in (16) is interpreted as exemplification. Steinbrenner avoids deciding on an interpretation of "refers to" and thereby he avoids the problem of circularity without getting rid of it. It would be eliminated if (16) was meant as introducing an undefined notion, similar to (E). At the beginning of his paper (Steinbrenner 2005, 228), Steinbrenner gives a hint in this direction when he points out that Goodman does not intend to define "denotation" and "exemplification" (with reference to $M M$, 55, where Goodman makes such a claim for "reference" only). But later on he explicitly treats (16) as a definition (Steinbrenner 2005, 230, note 5).

Perhaps Steinbrenner is worried by another threat of circularity. Indeed he writes that one may suspect a circularity "because Goodman seemingly wants to define the term 'reference' with the help of the terms 'denotation' and 'exemplification', but at the same time cannot explain the term 'exemplification' without the help of the terms 'denotation' and "reference" (Steinbrenner 2005, 228). This suggests that the circularity is not generated by the definition of "exemplification" alone but by its interplay with the definition of "reference"; it would result because "reference" is defined in terms of exemplification, which is defined in terms of reference. But Steinbrenner's proposal cannot avoid this circularity because in (16) "exemplification" is still defined with the help of "refers to". It is no surprise that Steinbrenner does not eliminate this circularity since, as he rightly insists, there is no such circularity because Goodman does not intend to define "reference" (Steinbrenner 2005, 228). 
Sometimes, however, Steinbrenner seems to think that circularity might arise since Goodman characterizes "reference" with the help of two terms while explaining one of them ("exemplification") in terms of the other ("denotation") (cf. Steinbrenner 1996, 85f). His proposal is intended to solve this problem by keeping denotation out of (16). However, it is not easy to see what the circularity should be, and even less how it could be eliminated by resorting to (16) since, not surprisingly, Steinbrenner explains "extension" (in connection with his characterization of systems) with the help of "denotation" (cf. Steinbrenner 1996, 89; 2005, 231).

All in all, it seems that, despite his own announcements, Steinbrenner is not so much trying to avoid circularity but to make sure that exemplification cannot be confused with denotation at least in theory (cf. Steinbrenner 2005, 231). For this purpose, he proposes to rely on a presupposed denotational system and the difference it introduces between labels and denoted objects, which in turn may serve as samples. But this would amount to characterize denotation and exemplification in terms of labels and samples instead of the other way around. ${ }^{19}$

\section{Conclusion}

Besides the last two accounts that not only fail but are also unnecessary as ways out, we have discussed four proposals to interpret the two conditions for exemplification Goodman formulates in (1). According to our proposal (E), "exemplification" is a basic notion, and hence undefined; the other alternatives suggest various definitions. (D) defines "exemplification" as denotation in both directions, (RD) as unqualified reference which presupposes denotation in the opposite direction and (R) takes "reference" as the only basic notion and defines "denotation" as unidirectional and "exemplification" as bidirectional reference.

${ }^{19}$ There is a similar tendency in $(L A, 58)$. But at this point Goodman is not dealing with the semantical conditions for denotation and exemplification, but with the epistemological criteria that might be used for deciding in specific cases whether a given reference is an instance of denotation or of exemplification. 
We argued that (D) as well as (R) fail from both an exegetic and a systematic point of view. Goodman unambiguously makes clear, firstly, that exemplification is a nondenotational form of reference and consequently cannot be defined in terms of denotation as in (D); and, secondly, that denotation in the same direction is not a necessary condition for exemplification as (D) and (R) imply. Both suggestions are also materially inadequate since they face all three of Dempster's objections. They obscure the distinction between exemplification and denotation and consequently between samples and labels, they turn exemplification into a symmetric relationship (which is implausible) and they are subject to countless counter-examples. For (RD) the situation seems, at least exegetically, to be less clear. There are frequent formulations in Goodman's writings stating that exemplification is being denoted (or possession) plus reference; if those statements could be read as definitions, (RD) would result directly. But Goodman never characterizes any of his formulations as a definition of "exemplification" and there is not even a passage which makes us expect such a definition. From an exegetic point of view, (RD) is therefore at least not inevitable. And it fails from a systematic point of view since it is subject to the same counter-examples as (D) and (R). Hence, only proposal (E) remains. The price for accepting (E) is that Goodman's theorems (b) and (c) have to be weakened and (1.1) has to be replaced by (1.2) or (1.3). Consequently, in statements that claim that exemplification is being denoted (or possession) plus reference either "reference" has to be interpreted as exemplification or the "is" as a conditional. Elgin does the second in her "official" introduction of the term and nowhere formulates Goodman's problematic theorems. Thus, while our suggestion (E) is at least defensible as an interpretation of Goodman, it is highly plausible as an interpretation of Elgin. The price for accepting (E) is well worth paying since $(\mathrm{E})$ is the only proposal not challenged by the counter-examples. It is thus the only materially adequate reconstruction of Goodman's notion of exemplification. 


\section{References}

Beardsley, M. (1975): "Semiotic Aesthetics and Aesthetic Education", in: Journal of Aesthetic Education 9 (1975), 5-26.

Beardsley, M. (1978): “'Languages of Art' and Art Criticism”, in: Erkenntnis 12 (1978), 95-118.

Carnap, R. (1971) [1950/62]: Logical Foundations of Probability, London: University of Chicago Press; Routledge and Kegan Paul, 1971.

Coldron, J. (1982): "Peltz on Goodman on Exemplification", in: Journal of Aesthetic Education 16 (1982), 87-93.

Dempster, D. (1989): "Exemplification and the Cognitive Value of Art", in: Philosophy and Phenomenological Research 49 (1989), 393-412.

Elgin, C. Z. (1983): With Reference to Reference, Indianapolis; Cambridge: Hackett, 1983.

Elgin, C. Z.(1993): "Relocating Aesthetics: Goodman's Epistemic Turn", in: Revue Internationale de Philosophie 46 (1993), 171-186. Reprinted in Elgin, C. Z.: Between the Absolute and the Arbitrary, Ithaca; London: Cornell University Press, 1997, 63-80; and in Elgin, C. Z. (ed.): Nelson Goodman's Philosophy of Art, New York: Garland, 1997, (= The Philosophy of Nelson Goodman. Selected Essays. Vol. 3), 1-17.

Elgin, C. Z.(1995): "Metaphor and Reference", in: Radman, Z. (ed.): From a Metaphorical Point of View. A Multidisciplinary Approach to the Cognitive Content of Metaphor, Berlin; New York: de Gruyter, 1995, 53-72.

Elgin, C. Z.(1996): Considered Judgment, Princeton; New Jersey: Princeton University Press, 1996.

Elgin, C. Z.(ed.) (1997a): Nelson Goodman's Theory of Symbols and Its Applications, New York: Garland, 1997 (= The Philosophy of Nelson Goodman. Selected Essays. Vol. 4).

Elgin, C. Z.(1997b): "The Power of Parsimony", in: Philosophia Scientiae 2 (1997), 89-104.

Goodman, N. (1975): [Letter to Monroe Beardsley], in: Journal of Aesthetic Education 9 (1975), 25-26. 
Heydrich, W. (1993): “A Reconception of Meaning”, in: Synthese 95 (1993), 77-94 (Reprinted in: Elgin 1997a, 155-172).

Martin, R. (1981): "On Some Aesthetic Relations", in: Journal of Aesthetics and Art Criticism 39 (1981), 258-264.

Peltz, R. (1972): "Nelson Goodman on Picturing, Describing, and Exemplifying", in: Journal of Aesthetic Education 6 (1972), 71-86.

Steinbrenner, J. (1996): Kognitivismus in der Ästhetik, Würzburg: Königshausen \& Neumann, 1996.

Steinbrenner, J. (2005): "Exemplifikation und Bezugnahmefeld", in: Steinbrenner, J.; Scholz, O., R.; Ernst, G. (eds): Symbole, Systeme, Welten. Studien zur Philosophie Nelson Goodmans, Heidelberg: Synchron, 2005, 227-234.

Textor, M. (2008): "Samples as Symbols", in: Ratio 21 (2008), 344-359. 